\title{
Demonstration of the ASTRAL post accidental code
}

\author{
C. Mourlon and G. De Drouas
}

Institute of Protection and Nuclear Safety (IPSN), Division of Environmental Protection (DPRE),
Laboratory of Environmental Modelling (LMODE),
CE Cadarache, 13108 Saint-Paul-lez-Durance cedex, France

\begin{abstract}
Whether for studying impacts of a hypothetical nuclear accident or for handling a crisis situation, experts and decision-makers, after a large scale atmospheric radioactive release, want to assess the contamination of the environment and food products, derive from it the potential exposure of populations to ionized radiations, predict the evolution of the situation and propose different scenarios for the management of contaminated zones. Starting from estimations or measurements of a radionuclide deposit on the ground, the ASTRAL computer code meets these demands : it calculates the evolution of concentrations in the food chain components and the doses to man due to external exposition and internal intake through inhalation of resuspended radionuclides and ingestion of contaminated foodstuff. Furthermore, various counter-measures can be simulated and therefore the efficiency of different managements of contaminated zones and products can be evaluated. A first version of ASTRAL has been released in 1997, and is installed in IPSN's Crisis Technical Center and in EDF (Electricité De France). A demonstration of its second version, which is to be released at the end of year 2001, is proposed. For this version, the choice of an internet architecture was made : it enables quick viewing of results, intuitive ergonomics - thus requiring a minimized training - and facilitates the spreading and the maintenance of the software, since only a web browser is necessary for the customer.
\end{abstract}

\section{INTRODUCTION}

Should an important release of radionuclides occur in the environment, one would quickly have to assess radionuclide concentration in media and foodstuff, deduce the potential exposition of concerned populations to the radiations, predict the evolution of the situation and propose different scenarios of managing the contaminated zones. To achieve this, the ASTRAL code was created, which can be used by a relatively large group of people composing crisis centers or making predictive impact studies.

The starting point of the assessments is the ground deposit of radionuclides : the atmospheric dispersion phase and the cloud exposure are not taken into account here. The calculations achieved concern time evolution of radionuclide concentration in elements of the food chain as well as assessment of doses due to external and internal exposure. These values are compared to regulation limits and/or intervention levels. Different simulations of contaminated zones management can be led by applying counter-measures, dispositions meant to reduce the accident's impact on environment and populations.

The radionuclides taken into account are some of those met in case of accidents on a Pressurized Water Reactor (PWR) - Sr89, Sr90, Ru103, Ru106, I131, Cs134 and Cs137 - as well as transuranic actinides - Am241, Cm242, Cm244, Np237, Np239, Pu238, Pu239, Pu240 and Pu241.On a temporal point of view, the predictions extend to 3 years after the deposition.

After two years of functioning, the user feedback raised a several points that could be improved, as well as new functionalities that could be added. The developing of a second version has therefore been undertaken following a Quality Assurance program. Currently being tested the new code should be launched by the end of year 2001 ; this nearly finished tool is demonstrated at the ECORAD Congress.

\section{CALCULATIONS PERFORMED}

The environment studied comprehends the agricultural ecosystem and, in the new version, the forest ecosystem. The calculations performed are radionuclide concentrations in different products of these two ecosystems and doses to man with different exposure scenarios and for different age groups (detailed information in IPSN report for ASTRAL V2 [1]).

For the agricultural ecosystem, different food types refer to different equation sets. For plants, three types are distinguished : annual crops, market garden produce and pasture grass. For animal products, contamination being attributed to ingestion, concentration is derived from that of the plants, using daily intakes defined by feeding calendars. For the forest ecosystem, three food types refer to different equation sets : mushrooms, berries and game. The radionuclide concentrations and their time evolution are then 
compared to maximum permitted levels (figure 1). The default values for the limits are those of the Euratom regulation, but can as well be any limit required by the decision maker.

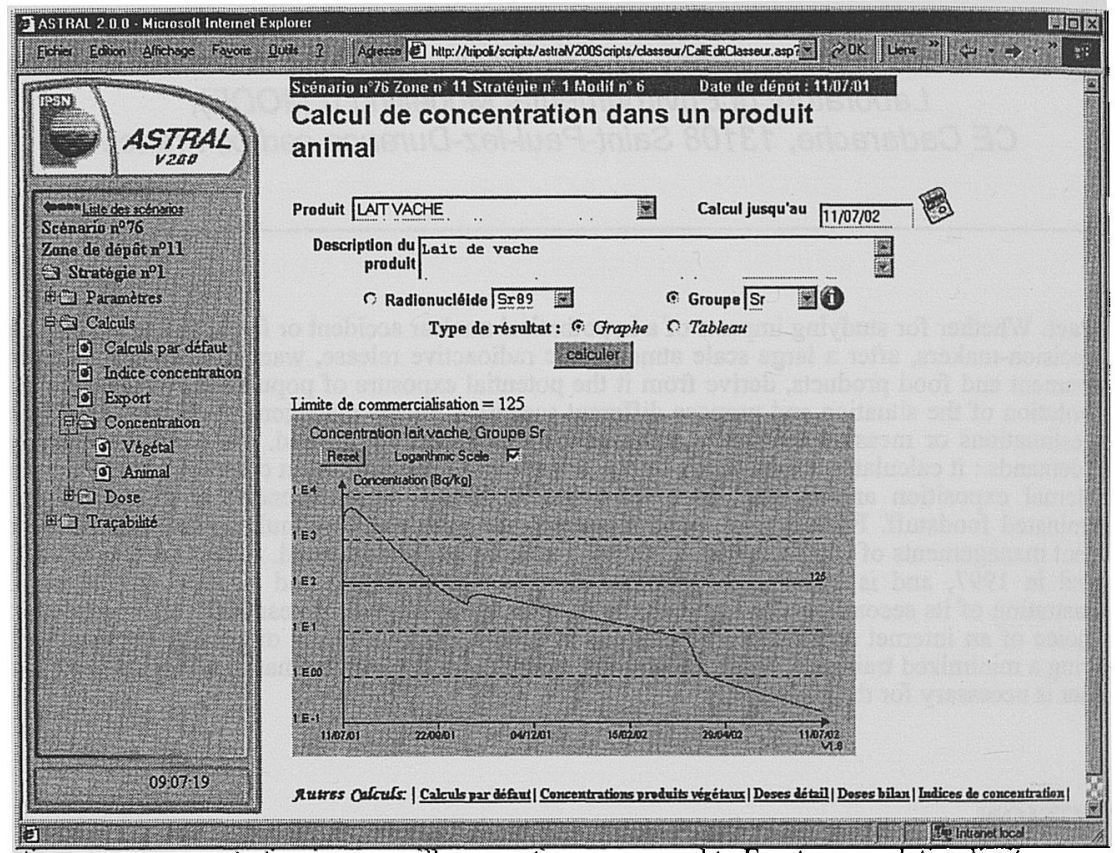

Figure 1: Srontium group concentration in cow milk versus time, compared to Euratom regulation limit.

The exposure pathways of man are external exposure to deposits, inhalation of resuspended particles, and ingestion of contaminated foodstuff. The accidental phase doses - due to cloud shine or inhalation in the plume - are not considered. The doses calculated in ASTRAL day by day or on a user defined time period can be effective doses or thyroid doses, in the case of contamination (inhalation and ingestion). Nine exposure scenarios are proposed for dose calculations, reflecting six age groups - as defined in the ICRP 72 [2]- and different lifestyles such as rural dwellers, hunters, berry and mushroom gatherers and forest workers. Most parameters, such as the diet, contaminated fraction of foodstuff or time spent in different environments, can be modified by the user, enabling him to create his own exposure scenario. The results are presented as tables and pie-charts, making easier the sighting of critical exposure pathways, radionuclides and foodstuffs (see figure 2).

While in a first stage calculations are performed to evaluate the evolution of the situation with :0 human intervention, it is possible that in future stages, one will want to see how the initial situation evolves with different ways of handling it. Diverse counter-measures can be simulated in ASTRAL, some aimed at reducing concentrations of foodstuff, others at limiting exposition of man :

- for agricultural plants : use of fertilizers and/or lime, deep ploughing, top soil layer stripping;

- for agricultural animal products : use of Prussian blue or clay, change in feeding - especially interruption of grazing;

- for man : change in the food intake, ban of consumption of local products, modification of time budgets in different environments...

By comparing the levels reached by concentrations and doses in these various cases, the efficiency of different managements of contaminated zones can thus be evaluated. 


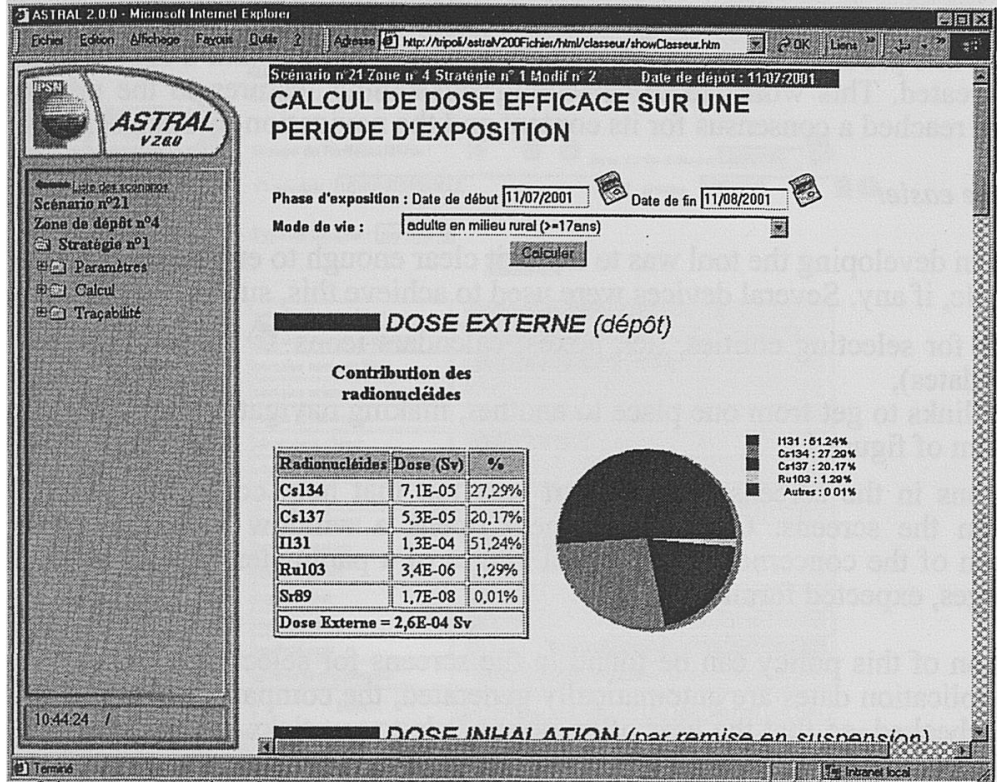

Figure 2 : Example of dose presentation for a given time period and individual type.

\section{CHARACTERISTICS OF THE SECOND VERSION}

The development of this version started in 1999 and has been subject to a quality assurance program. The identified customers and users have been associated in a control committee expressing needs, validating choices and checking the good progress of the project. The programming is currently being validated by test scenarios that check the equations, parameters, functionalities and navigation through the screens.

\subsection{New technology and architecture}

The technology and architecture chosen for the second version of ASTRAL are different from the previous ones. It now uses an internet technology and is of a three-third type, the software requiring an Oracle database, a Microsoft web server and a consultation station. These choices offer important advantages:

- the spreading and maintenance of the tool are very easy. In fact, once the server and the database have been installed on a network, the users only need a web browser to run the code. The initial installation and the updating are thus no more to be made on every user station, but only on the server and/or the database.

- the code may be installed on a portable computer, which hosts the database, the internet server and the web browser. This enables working isolated from the network, on the field or in decision making sites.

- the access to results is much quicker, for they are no longer all systematically calculated and saved. Only those required are calculated or saved, on decision of the user.

\subsection{New scientific features}

The second version integrates a new module dedicated to the forest ecosystem, which has been adapted from the one developed by IPSN in collaboration with the Finnish Nuclear Safety Authority STUK [3]. Thyroid dose calculations are added. Finally, the list of radionuclides dealt with is extended by taking into account the transuranics actinides mentioned earlier, and more exposure scenarios are proposed with the integration of the forest module and the introduction of six age groups.

\subsection{Improved ergonomics}

As for any radioecological tool, a large number of parameters intervene in the calculations. All parameters can be modified, even though different user profiles grant different rights in handling them. Also, to define his request, the user must chose many input data (radionuclide, foodstuff, lifestyle, 
dates...). As a result of this, radioecological tools can easily end up with abundant and complicated screens. To avoid this problem, special care was given to ergonomics and a user group, charged to test a prototype, was created. This work led to correcting and adding features to the prototype : after a few sessions, the tool reached a consensus for its content and the navigation through screens.

\subsubsection{Making use easier}

One of the goals in developing the tool was to make it clear enough to enable people to use it with as little training as possible, if any. Several devices were used to achieve this, such as :

- list boxes for selecting entities, tick boxes, calendars icons (to avoid format problems when inputting dates),

- hypertext links to get from one place to another, making navigation through the screens very easy (see bottom of figure 1).

- help buttons in the screens. Wherever it was felt that help could be needed, $\curvearrowright$ buttons were inserted in the screens. Clicking on them opens a window giving additional information: description of the concerned entity, usual values of a parameter, default value origin, authorized value ranges, expected format ...

An illustration of this policy can be found in the screens for selecting counter-measures. Whenever possible, their application dates are automatically generated, the compatibility between counter-measures is automatically checked, so that the user often only needs to put ticks in boxes. These screens use most of the pre-mentioned devices as shown in figure 3 .

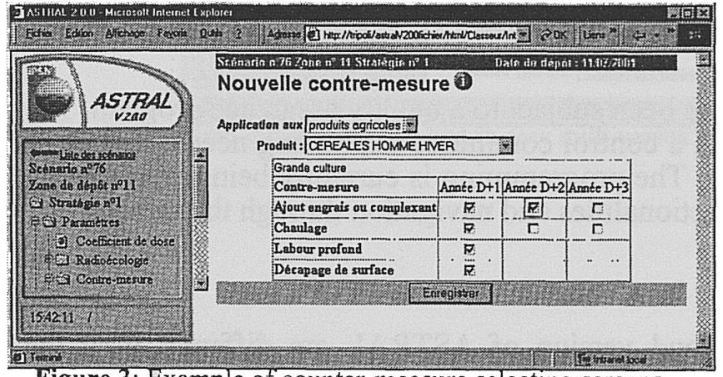

Figure 3: Example of counter-measure selecting screens.

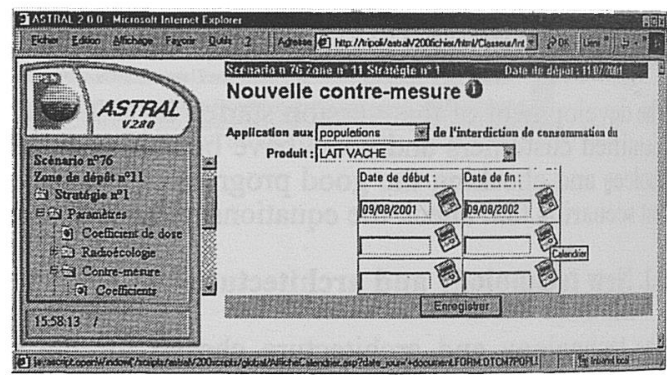

\subsubsection{Making result interpretation and comprehension easier}

Whenever possible, the default presentation of results is a graph, but the possibility is always given to shift to a table presentation. Graphs are meant to be synthetic; however precise information must remain reachable. Therefore small applications have been developed : the user can zoom in on parts of the graphs, getting the detailed information of values and dates in a dedicated information box. He may also shift from linear to logarithmic scales by using a click box (see figure 1).

ASTRAL can be used in crisis situations, in which case its results are intended to be communicated to decision makers, usually people not belonging to the scientific world. The information thus cannot just consist of values such as $\mathrm{Bq} / \mathrm{kg}$ concentrations for foodstuff : results must be understandable and interpretable by non-specialists and must always be compared to some limit. An example of the efforts deployed to make information clear and communicable is the ratio of the concentration to the limit : it is allocated to a group depending on whether it is smaller than 30\% (green color assigned), between 30\% and $300 \%$ (orange) or bigger than $300 \%$ (red). This processing of the information, with the use of colors for each group, enables a quick evaluation of the products and time periods where problems will be encountered and may help to prioritise decisions and counter-measures (see figure 4). 


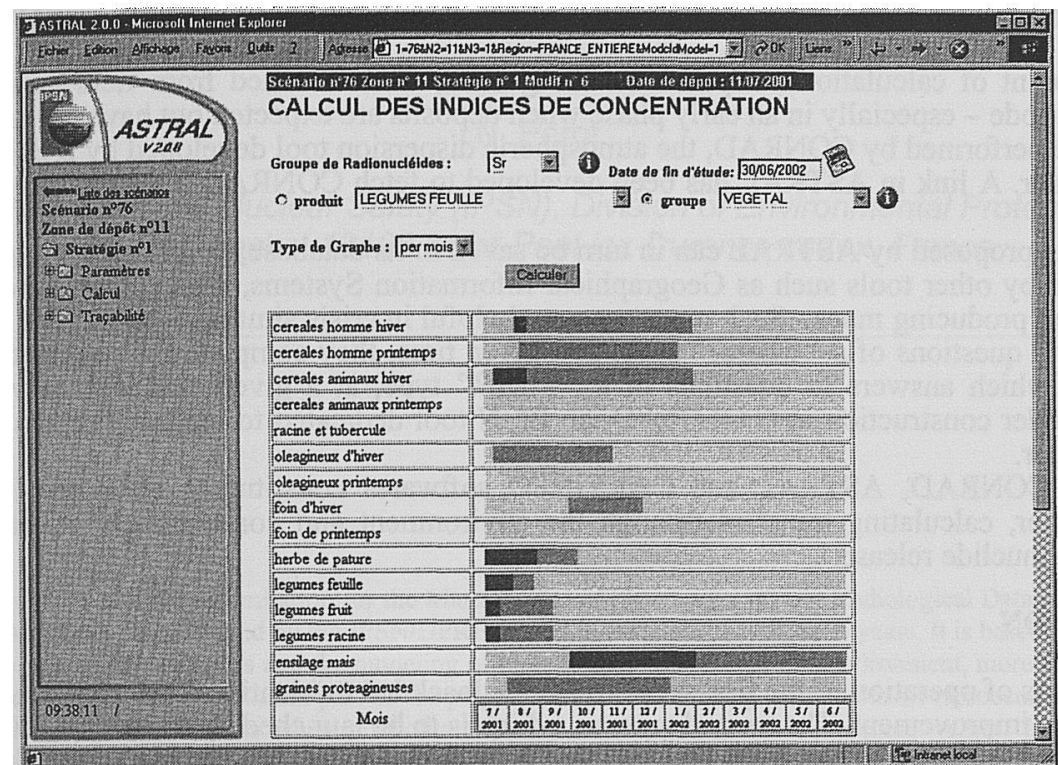

Figure 4: Time evolution of the concentration/limit ratio, displayed with three colors depending on its value : enabling a quick identification of problematic products and time periods.

\subsubsection{Default results}

The tool enables the calculation of a wide variety of results. However, the feedback from IPSN's participation in crisis exercises has shown that, in crisis situations, too dense information doesn't help decision makers and that the latter only want a few results - often the same. Therefore the endpoints that experience has taught to be most relevant or systematically asked for have been grouped in a specific result screen. This "default results" screen launches the following calculations :

- concentration in milk after the first day and at the day the maximum is reached, for each radionuclide group, using the three color convention for comparison to the limit ;

- concentration in leafy vegetables after the first day and after the first week, for each radionuclide group, using the three color convention for comparison to the limit ;

- doses after one day, one week, one month and one year for six individual types : baby (0-1 year), very young children (1-2 y), rural young children $(2-7 \mathrm{y})$, rural children (7-12 y), rural teenagers (12-17 y) and rural adults (above $17 \mathrm{y}$ ). For each of these time periods and individual types, the effective doses are calculated - for all radionuclides, all exposure pathways.

These results, to be used in a first phase of a crisis, present the state of the usually most sensible foodstuff (milk and leafy vegetables) and enable the identification of the most vulnerable individual types - as well as the related critical time periods.
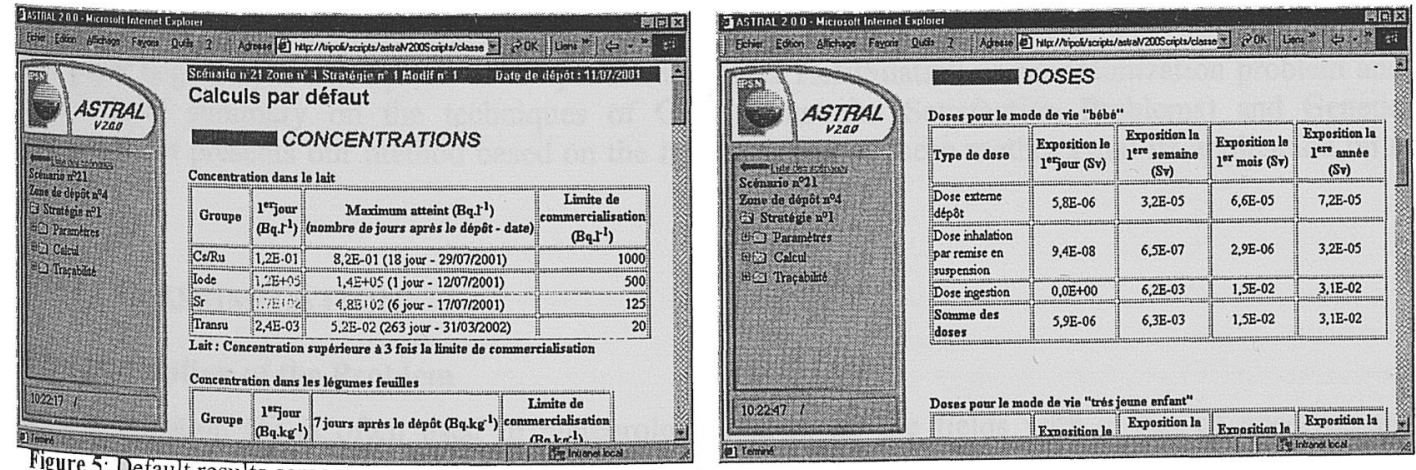

Figure 5: Default results screen. 


\subsection{Links with other tools}

The starting point of calculations, deposits on the ground, can be issued from field measurements or predicted by a code - especially in an early phase when deposits are expected but have not occurred. Such predictions are performed by CONRAD, the atmospheric dispersion tool developed by IPSN for its Crisis Technical Center. A link in ASTRAL has been developed to fetch CONRAD's results and input them in its database.

The results proposed by ASTRAL can in turn be saved in its database, on the user's request, in order to be exploited by other tools such as Geographical Information Systems, which enable crossing spatial information and producing maps. Such maps are very helpful in crisis situations to decision makers since by answering to questions of a "where?" type, at a given time, they complete the information delivered by ASTRAL, which answers to questions of a "when?" type, at a given site. This is why a link to ASTRAL is under construction in CARTINFO, an IPSN tool dedicated to mapping results for its Crisis Technical Center.

Thus the CONRAD, ASTRAL and CARTINFO softwares constitute a chain for IPSN's Crisis Technical Center, calculating transfers through the environment and consequences of an atmospheric accidental radionuclide release.

\section{CONCLUSION}

After a few years of operation of the first version, the feedback led to identification of new needs and points requiring improvement. The second version, which is to be launched by the end of 2001 , is the result of two years of work in constant consultation of customers and users through a control committee and a user group : the tool should therefore meet the various and sometimes conflicting demands -the needs for handling a crisis situation not being those for carrying impact studies, for example. It integrates some new scientific features (a forest module, additional radionuclides, additional age groups and thyroid dose calculations), but the main evolution lies in the architecture and internet technology chosen. The latter make the installation, spreading and maintenance of the tool much easier, make the tool very user friendly and enable gain in speed.

\section{References}

[1] Calmon P. And Mourlon C., Equations et paramètres du logiciel ASTRAL V2, (2001), report IPSN/DPRE/SERLAB n ${ }^{\circ} 01-19$ (to be published).

[2] International Commission on Radiological Protection. Age-dependent Dose to Members of the Public from Intake of Radionuclides: Part 5 Compilation of Ingestion and Inhalation Dose Coefficients, Publication 72, (Pergamon Press, Oxford, 1996).

[3] Calmon P. Etude des transferts des radionucléides au sein d'écosystèmes forestiers. 3ème partie : un modèle de transfert des radionucléides dans les forêts aux champignons, baies et gibiers, (1999), repott IPSN/DPRE/SERLAB n ${ }^{\circ}$ 99-020 (P). 